\title{
Low-Complexity Chromatic Dispersion Equalizer for 400G Transmission Systems
}

\author{
C. S. Martins ${ }^{1}$, S. B. Amado' ${ }^{1}$, S. M. Rossi ${ }^{2}$, A. Chiuchiarelli ${ }^{2}$, J. D. Reis ${ }^{2}$, A. Carena ${ }^{3}$, \\ F. P. Guiomar ${ }^{3}$ and A. N. Pinto ${ }^{1}$ \\ ${ }^{1}$ Department of Electronics, Telecommunications and Informatics, University of Aveiro and Instituto de \\ Telecomunicações, 3810-193, Aveiro, Portugal, csmartins@av.it.pt \\ ${ }^{2}$ CPqD, Division of Optical Technologies, 13086-902, Campinas - SP, Brazil, jacklyn@cpqd.com.br \\ ${ }^{3}$ DET, Politecnico di Torino, Corso Duca degli Abruzzi, 24, 10129, Torino, Italy, fernando.guiomar@ polito.it
}

\begin{abstract}
We experimentally demonstrate a reduced complexity time-domain CD equalizer in a dual-carrier 400G PM-16QAM system, yielding hardware savings of over 99\% in terms of multipliers, and a latency reduction of $\sim 80 \%$ over standard frequency-domain CD equalization.

OCIS codes: (060.2360) Fiber optics links and subsystems; (060.1660) Coherent communications.
\end{abstract}

\section{Introduction}

The recent advances in digital coherent optical transmission systems have enabled near-optimum compensation of linear propagation impairments, such as chromatic dispersion (CD) and polarization-dependent effects, through digital signal processing (DSP)-based algorithms [1]. Among these impairments, CD equalization (CDE) is a critical subsystem, since it is known to contribute to a large portion of the overall DSP complexity and power consumption [2].

In digital coherent optical systems, CDE can be performed either in time-domain (TD) or frequency-domain (FD). The standard TD equalization is performed by a finite-impulse response (FIR) filter, whose tap coefficients can be determined from the inverse CD transfer function [1]. In FD the equalization exploits the computational efficiency of fast Fourier transform (FFT) in conjunction with overlap-save/add algorithms [3]. Aiming to reduce implementation the complexity, several optimized CDE algorithms have been proposed, including the use of sub-band processing [4] and coefficient quantization [5,6]. Exploiting the high multiplicity of the quantized TD-CDE coefficients, we have recently proposed a distributive FIR-CDE (D-FIR-CDE) algorithm for 100G PM-QPSK transmission systems, which was shown to yield significant complexity and latency gains [6].

In this paper, we provide an experimental validation of the D-FIR-CDE algorithm in a dual-carrier 400G PM16QAM transmission system. Comparing its complexity with standard FD-CDE, we demonstrate hardware savings of over $99 \%$ in terms of multiplication operations together with a processing latency reduction over $80 \%$.

\section{Distributive FIR-CDE}

In time-domain, $\mathrm{CD}$ equalization can be performed using a complex-linear FIR filter,

$$
y(n)=\sum_{k=0}^{N-1} x(n-k) c(k),
$$

where $y(n)$ represents the equalized sample, $x$ are the received samples, $c$ are the complex FIR coefficients and $N$ is the number of FIR taps. In order to reduce the implementation complexity of the FIR-CDE we apply a quantization process to the set of FIR coefficients, $\{c(k)\}$, obtaining a new set of quantized values, $\left\{c^{Q}(k)\right\}$, given by,

$$
c^{Q}(k)=\frac{\left\lfloor\Delta \frac{c(k)}{\max \left(\left|c_{\mathrm{r}}(k)\right|,\left|c_{\mathrm{i}}(k)\right|\right)}\right\rfloor}{\Delta}, k=0, \ldots, N-1,
$$

where $\Delta$ is the quantization factor, $c_{\mathrm{r}}(k)$ and $c_{\mathrm{i}}(k)$ are the real and imaginary parts of $c(k)$, respectively, and $\lfloor\cdot 7$ represents the nearest integer operation [6]. From the inspection of the quantized coefficients, we can note that a given value of $\Delta$ generates a set with a maximum of $2 \Delta+1$ quantization levels (possible values), $\left\{c_{\mathrm{m}}\right\}$, for $c_{\mathrm{r}}^{Q}(k)$ and $c_{\mathrm{i}}^{Q}(k)$. Moreover, it is observed that each possible value, $c_{m}$, repeats over $c_{\mathrm{r}}^{Q}(k)$ and $c_{\mathrm{i}}^{Q}(k)$ with a given multiplicity (number of repetitions), which increases with decreasing $\Delta$ and increasing $N$. In this context, it is crucial to observe that the multiplicities over real and imaginary parts of $c(k), c_{\mathrm{r}}^{Q}(k)$ and $c_{\mathrm{i}}^{Q}(k)$, are much higher than that of the complex-valued coefficients, $c_{\mathrm{r}}^{Q}(k)+j c_{\mathrm{i}}^{Q}(k)$. In order to take maximum advantage from the distributive property of multiplication over addition, we can perform complex multiplications $(\mathrm{CMs})$ between $x(n-k)$ and $c^{Q}(k)$ as [6],

$$
x(n-k) c^{Q}(k)=x(n-k) c_{\mathrm{r}}^{Q}(k)+j x(n-k) c_{\mathrm{i}}^{Q}(k) .
$$




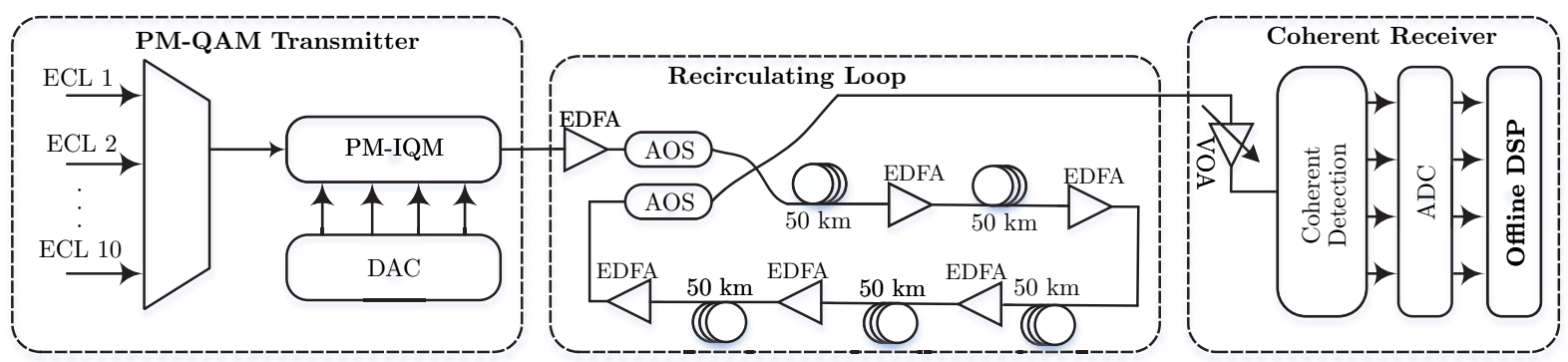

Fig. 1: Experimental setup of the 5-WDM transmission system composed of $2 \times 31.5$ Gbaud PM-16QAM 400G superchannels.

Each complex multiplication in the FIR-CDE equalizer is then divided into two multiplications between a complexvalued signal sample, $x(n)$, and the correspondent real and imaginary parts of the quantized coefficients $\left(c_{\mathrm{r}}^{Q}\right.$ and $\left.c_{\mathrm{i}}^{Q}\right)$. This enables to group the incoming signal samples according to the quantized values of their respective coefficients, thereby applying the distributive property of multiplication over addition to drastically reduce the overall number of multiplications. The resulting D-FIR-CDE filter architecture is detailed in Fig. 3 of [6]. Note that the proposed algorithm assumes a priori knowledge of the FIR coefficients, which can be the case for long-haul point-to-point optical links.

\section{Experimental Results}

In this section, we perform the experimental validation of D-FIR-CDE over a long-haul 400G transmission system. The laboratorial setup is depicted in Fig. 1. At the transmit-side, a $63 \mathrm{GSa} / \mathrm{s}$ DAC with $\sim 14 \mathrm{GHz}$ electrical bandwidth generates the analog I and Q components for both polarizations, which are Nyquist-shaped by a raised cosine filter with a roll-off factor of 0.1 . The optical carriers are generated by an array of 10 external-cavity lasers (ECL) with $100 \mathrm{kHz}$ linewidth, yielding 5 WDM superchannels, consisting of two PM-16QAM subcarriers modulated at 31.5 Gbaud. The transmission link is composed of a recirculating-loop, controlled by two acousto-optical switches (AOSs), with five 50$\mathrm{km}$ spans of ultra-low-loss fiber, characterized by $\alpha$ of $0.162 \mathrm{~dB} / \mathrm{km}$ and dispersion slope (D) of $20.2 \mathrm{ps} / \mathrm{nm} / \mathrm{km}$. Loss is recovered by EDFAs with 6-dB noise figure. At the receiver side, a variable optical attenuator (VOA) is utilized to adjust the optical power that reaches the photodiodes. After detecting the superchannel subcarriers, the electrical signal is sampled by a $80 \mathrm{GSa} / \mathrm{s}$ real-time oscilloscope with $34 \mathrm{GHz}$ electrical bandwidth and then offline post-processed. The DSP comprises CD and linear adaptive equalization, carrier recovery and bit-error ratio (BER) counting. For CDE, we have applied both standard FD-CDE and our advanced D-FIR-CDE algorithm [6].

\subsection{Assessment of the D-FIR-CDE Performance}

Figs. $2 \mathrm{a}$ and $2 \mathrm{~b}$ show the experimental results in terms of performance of D-FIR-CDE in comparison with the maximum performance achieved with standard FD-CDE. The number of coefficients is set to $~ 60 \%$ of its theoretical value and the FFT-block size is defined to obtain virtually zero performance penalty [1]. The evolution of BER as a function of transmission length shown in Fig. 2a indicates that D-FIR-CDE tends to become more robust to the quantization process as higher BER thresholds are considered, which in turn enables to decrease the quantization factor $\Delta$ without incurring into excessive performance penalty. As shown in Fig. $2 \mathrm{~b}$ for a fixed transmission length $L=4000 \mathrm{~km}$, the BER performance can be finely adjusted by the choice of $\Delta$, thus providing enhanced flexibility to tradeoff performance and computational effort, depending on the actual system margin to the established FEC limit. Considering a typical BER limit between $2.4 \times 10^{-2}$ and $2.7 \times 10^{-2}$, the quantization factor $\Delta$ can be adjusted between 4 and 8 , while ensuring compliance with the FEC threshold.

\subsection{Computational Effort Comparison Against FD-CDE}

In Figs. $2 \mathrm{c}$ and $2 \mathrm{~d}$ the complexity of D-FIR-CDE is compared with the widely used FD-CDE in terms of total number of real multipliers (RMs) and real adders (RAs). As a figure of merit we adopt the complexity reduction gain, $G_{R}$, in percentage, defined as $G_{R}=\left(1-O / O_{\text {ref }}\right)$, where $O$ and $O_{\text {ref }}$ are the number of operations required by D-FIR-CDE and FD-CDE, respectively. The total number of operations for D-FIR-CDE and FD-CDE are calculated according to the analytical formulation derived in [6]. In order to assess the impact of carrier symbol-rate of 400G superchannels on CDE complexity, we also extended the computational effort analysis to symbol-rates ranging from 4 GBaud (16 carriers) to 64 GBaud (single-carrier) per carrier. It is important to emphasize that the analysis performed here always refers to the overall complexity required by the $n$-carrier $400 \mathrm{G}$ system. For each symbol-rate we determine the correspondent number of taps, $N$, and the FFT block-size is defined as the minimum value that yields penalty-free equalization. The degree of parallelization is also adjusted for each symbol rate considering a reference DSP clock frequency of $500 \mathrm{MHz}$. 

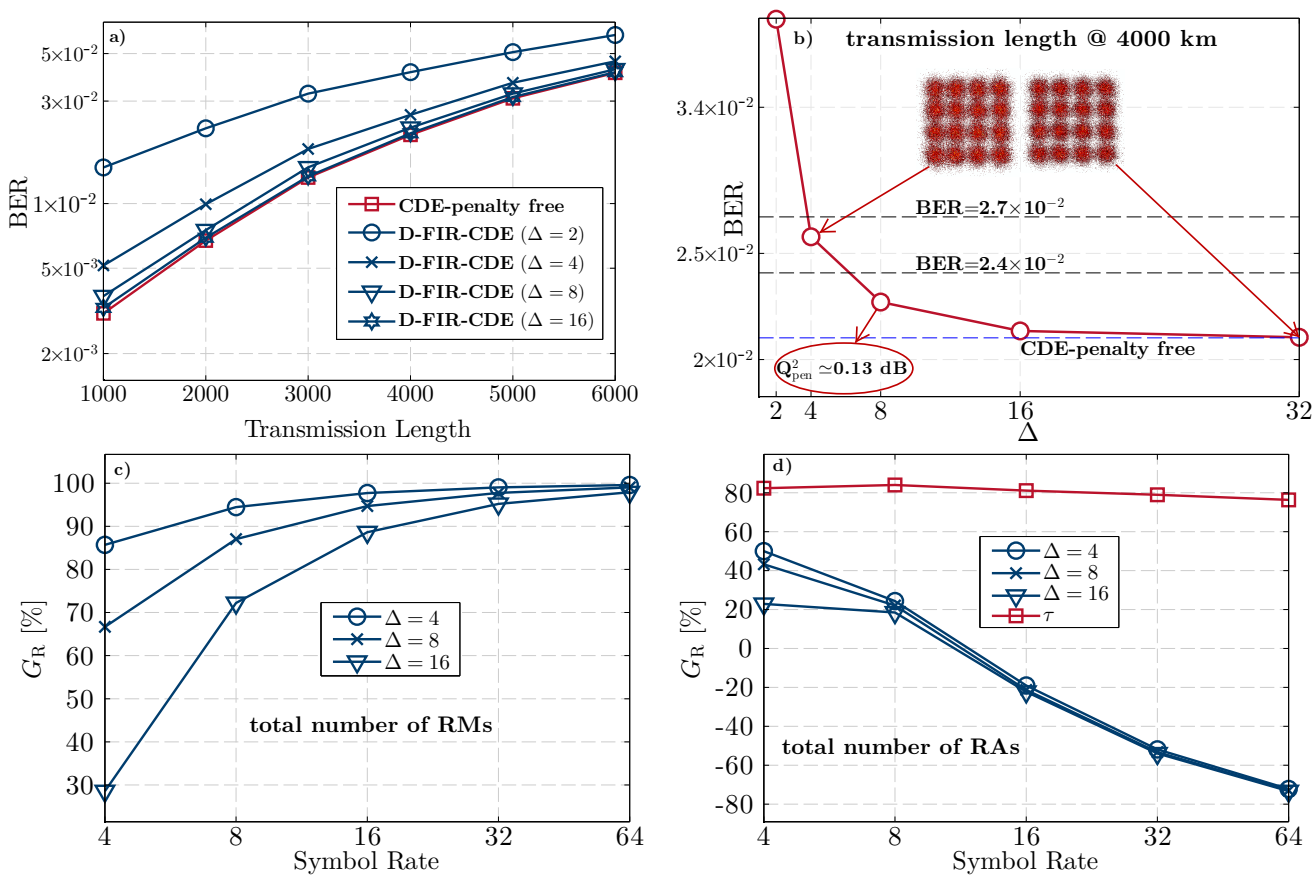

Fig. 2: a) and b) Performance of D-FIR-CDE depending on the transmission length (a) and on the quantization factor, $\Delta$, for $L=4000 \mathrm{~km}$ (b); c) and d) Hardware savings in terms of RMs (c) and RAs and latency (d) of D-FIR-CDE over FD-CDE, as a function of symbol rate.

Fig. 2c reveals increasing efficiency of D-FIR-CDE in terms of total number of RMs with the increase of symbol rate, reaching more than $\sim 99 \%$ gain for the single-carrier 64 GBaud $400 \mathrm{G}$ solution. Also note that higher symbolrates enable the use of larger quantization factors, $\Delta$, without significantly reducing the D-FIR-CDE efficiency. This gain in terms of RMs comes at the expense of an increasing number of RAs with increasing symbol-rate per carrier. Indeed, at 64 GBaud the FD-CDE was found to require 70\% less RAs than the D-FIR-CDE. Nevertheless, these gains are highly compensated by the D-FIR-CDE advantage in terms of RMs, which is known to be key computational effort and power consumption indicator for practical real-time implementation [2]. In addition, Fig. 2d also shows the lower latency of D-FIR-CDE, which is enabled by its time-domain processing and can be over $80 \%$. Considering the experimental case study of a $2 \times 32$ GBaud $400 \mathrm{G}$ superchannel and a conservative quantization factor of $\Delta=8$, the achieved hardware savings in terms of RMs is of $\sim 99 \%$, while $\sim 50 \%$ more RAs are required.

\section{Conclusions}

Using a 400G $(2 \times 32$ GBd) PM-16QAM signal, we have experimentally demonstrated a low complexity timedomain CD compensation algorithm for digital coherent receivers. Relatively to the benchmark FD-CDE equalizer, D-FIR-CDE has revealed a much higher efficiency in terms of number of multipliers ( $99 \%)$ and processing latency $(\sim 80 \%)$. By adjusting the coefficient quantization factor, $\Delta$, the proposed equalizer provides an enhanced flexibility for the tradeoff between performance and complexity, depending on the system margin. Finally, extending the complexity analysis to other $400 \mathrm{G}$ configurations, we have demonstrated that the D-FIR-CDE algorithm can be an attractive solution for high symbol rate transmission, tackling the increasingly high complexity of CD compensation in such systems.

This work was supported in part by Fundação para a Ciência e a Tecnologia (FCT) through national funds, and when applicable co-funded by FEDER-PT2020 partnership agreement, under the project UID/EEA/50008/2013 (action SoftTransceiver), by the Ph.D. grant PD/BD/113817/2015 and by the European Commission through a Marie Skłodowska-Curie individual fellowship, project Flex-ON (653412).

\section{References}

[1] S. J. Savory, "Digital filters for coherent optical receivers," Opt. Express, vol. 16, no. 2, pp. 804-817, Jan 2008.

[2] B. Pillai et al., "End-to-end energy modeling and analysis of long-haul coherent transmission ...", JLT, vol. 32, no. 18, pp. 3093-3111, 2014.

[3] T. Xu et al., "Frequency-domain chromatic dispersion equalization using overlap-add methods ...", JOCN, vol. 32, no. 2, pp. 131-135, 2011.

[4] M. Taylor, "Compact digital dispersion compensation algorithms," Proc. OFC, paper OTuO1, 2008.

[5] A. Sheikh et al., "Dispersion Compensation FIR Filter with Improved Robustness to Coefficient Quantization Errors", pre-print JLT, 2016.

[6] C. S. Martins et al., "Distributive FIR-Based Chromatic Dispersion Equalization for Coherent Receivers", pre-print JLT, 2016. 